\title{
ANÁLISE DAS CARACTERÍSTICAS HIDROLÓGICAS DE TRÊS SUB-BACIAS DO RIO CARAPÁ (CANINDEYÚ, PARAGUAI) EM FUNÇÃO DAS MUDANÇAS DA COBERTURA VEGETAL
}

\author{
César Daniel Riveros Reys ${ }^{1}$, Nivaldo Eduardo Rizzi ${ }^{2}$, Hideo Araki ${ }^{3}$ \\ ${ }^{1}$ Eng. Agrônomo, M.Sc., Faculdad de Ciencias Agrárias, UNA, Asunción, Paraguai - cesarriveros_1972@ yahoo.com.br \\ ${ }^{2}$ Eng. Florestal, Dr., Depto. de Engenharia e Tecnologia Florestal, UFPR, Curitiba, PR, Brasil - niva@ufpr.br \\ ${ }^{3}$ Eng. Cartógrafo, Dr., Depto. de Geomática, UFPR, Curitiba, PR, Brasil - araki@ufpr.br
}

Recebido para publicação: 25/02/2010 - Aceito para publicação: 07/12/2010

\begin{abstract}
Resumo
O objetivo deste trabalho foi analisar o comportamento hidrológico em três sub-bacias da bacia hidrográfica do rio Carapá, localizadas no Departamento de Canindeyú, Paraguai, em 1985, 1999 e 2007, através de análise multitemporal do uso do solo e análise da resposta hidrológica pelo método de Curva Número com ênfase no parâmetro de coeficiente de escoamento superficial (CE). A metodologia de estudo foi dividida em duas etapas: classificação dos usos do solo e análise das mudanças da vegetação nativa e análise das classes geradas com adição de tipologias de solos para gerar os parâmetros hidrológicos nas três condições de umidade antecedente: normal (NII), seco (NI) e próximo da saturação (NIII). Os resultados indicaram diminuição da cobertura florestal nas três subbacias. Das três, o coeficiente de escoamento superficial nas três situações de umidade antecedente da sub-bacia 49, em 1985 e 1999, foi a mais alta $(\mathrm{NI}=6,42 ; \mathrm{NII}=30,88$; $\mathrm{NIII}=57,86)$ e a que indica maior possibilidade de degradação. No período de 2007, o coeficiente de escoamento superficial nas três situações de umidade antecedente da sub-bacia 01 foi a mais alta $(\mathrm{NI}=17,03$; $\mathrm{NII}=45,18$; $\mathrm{NIII}=69,32$ ), indicando maior possibilidade de degradação nessa sub-bacia por conta da ação da erosão hídrica.

Palavras-chave: Bacia hidrográfica; análise multitemporal; curva número; escoamento superficial.
\end{abstract}

\begin{abstract}
Analysis of hydrologic characteristics of three sub-basins of Carapa River basin (Canindeyú, Paraguay) in relation to changes of plant cover. The objective of this study was to analyze the hydrological behavior in three sub-basins of the river basin, Carapa, located in the Department of Canindeyú, Paraguay in 1985, 1999 and 2007 through multitemporal analysis of land use and hydrologic response analysis method Curve Number with emphasis on parameter runoff coefficient (EC). The methodology was divided into two steps: classification of land use and analysis of changes in vegetation and analysis of the generated classes with the addition of soil types to generate the hydrological parameters in the three antecedent moisture conditions: normal (NII) cleaning (NI) and close to saturation (NIII). The results showed decrease in forest cover in the three sub-basins. From the three parameters, the runoff coefficient in three different moisture history of the sub-basin 49 between 1985 and 1999 was the highest $(\mathrm{NI}=6.42, \mathrm{NII}=30.88, \mathrm{NIII}=57.86)$ and indicates a higher possibility of degradation. During 2007, the runoff coefficient in three different moisture history of the sub-basin 01 was the highest $(\mathrm{NI}=17.03$, NII $=45.18, \mathrm{NIII}=69.32)$, indicating a greater possibility of degradation the sub-basin due to the action of water erosion.

Keywords: Hydrographic basin; multitemporal analysis; curve number; runoff.
\end{abstract}

\section{INTRODUÇÃO}

A diminuição da superfície florestal no Departamento de Canindeyú, Paraguai, é uma realidade que gera preocupação constante na comunidade, pela falta de critérios para a realização de um aproveitamento racional dos recursos florestais. Ao longo dos anos, as áreas de florestas têm sofrido com a depredação e mudança de uso por parte de proprietários que optam, num primeiro momento, por derrubar a floresta primária para o aproveitamento da madeira e depois utilizarem essas áreas 
remanescentes para culturas anuais ou pastagens. A degradação acelerada do solo decorrente da mudança de uso fatalmente afeta a qualidade dos recursos hídricos e impacta negativamente na resposta hidrológica da bacia.

O objetivo deste trabalho foi analisar o comportamento hidrológico de três nascentes, denominadas como sub-bacias, da bacia hidrográfica do rio Carapá, localizada no Departamento de Canindeyú, Paraguai, nos anos de 1985, 1999 e 2007, através de análise multitemporal do uso do solo e análise da capacidade de armazenamento e resposta hidrológica da bacia.

\section{REVISÃO BIBLIOGRÁFICA}

A bacia hidrográfica pode ser definida como a área de captação natural da água da precipitação, drenando essa água por ravinas, canais e tributários, para um curso de água principal, tendo a vazão uma única saída, desaguando em um curso de água maior, lago ou oceano (TONELLO, 2005). Tucci et al. (1993) consideram a bacia hidrográfica como um sistema físico aberto, no qual a entrada é o volume de água precipitado e a saída é o volume de água escoado pelos canais escoadouros que carregam materiais provenientes da própria bacia, considerando-se como perdas intermediárias os volumes evaporados e transpirados, além daqueles que são infiltrados, permanecendo no solo ou migrando para as partes mais profundas. O fluxo de saída é determinado pelas características geológicas, geomorfológicas, pedológicas, de vegetação e do clima que incide na área.

Cardozo et al. (2006) manifestam que a vegetação presente na bacia, o solo e a água encontramse em interação permanente e dinâmica que responde diretamente às mudanças e interferências, sejam elas naturais ou de origem antrópica, que afetam o ecossistema como um todo. Os recursos hídricos constituem os indicadores das condições dos ecossistemas e refletem os efeitos de desequilíbrio das interações dos componentes da bacia hidrográfica. Para Chiaranda (2002), as funções fundamentais da bacia hidrográfica sofrem alterações, e quando a modificação do uso da terra dá-se em grandes extensões de área na bacia hidrográfica, podem ser observados impactos no comportamento do escoamento.

O escoamento superficial é um dos componentes do ciclo hidrológico, junto com a precipitação, interceptação, infiltração, transpiração e evaporação. É impulsionado pela gravidade, ultrapassando a barreira do atrito com a superfície do solo até se concentrar nas cotas mais baixas (VILLELA; MATTOS, 1975; TUCCI et al., 1993). Villela; Mattos (1975) acrescentam que o escoamento superficial sofre a influência de diversos fatores que ajudam ou prejudicam a sua ocorrência. Podem ser de natureza climática, que são as relacionadas com a precipitação, ou podem ser de natureza fisiográfica, referentes às características físicas da bacia (área, forma, permeabilidade, capacidade de infiltração e topografia da bacia). Tucci et al. (1993) ressaltam que a presença de vegetação na superfície do solo obstrui o escoamento superficial e favorece a infiltração em percurso. Da mesma forma, a vegetação provoca uma diminuição da energia cinética de impacto das gotas de chuva na superfície do solo, fator este que minimiza a erosão.

As mudanças originadas nas componentes biofísicas da superfície terrestre apresentam variações muito dinâmicas ao longo do tempo. Nesse sentido, as pesquisas relacionadas à coleta de dados através de sensoriamento remoto ou de cartografia muitas vezes conflitam com bancos de dados desatualizados, comprometendo a precisão dos mesmos. Nesse contexto, houve um esforço para desenvolver técnicas para detectar as mudanças ocorridas ao longo do tempo, utilizando-se ferramentas do sensoriamento remoto (JENSEN, 1996). Da mesma forma que se pode manipular um conjunto de imagens em diferentes bandas espectrais, podem-se manipular imagens de uma mesma banda em diferentes épocas. A essa manipulação de dados de diferentes épocas denomina-se análise multitemporal (NOVO, 1992).

Segundo Ferrer Juliá et al. (2003), o modelo de Curva Número foi desenvolvido pelo Serviço de Conservação de Solos (SCS), atualmente denominado Natural Resources Conservation Service (NRCS), dos EUA, durante a década de 50, para sua aplicação em todo o país. Chow; Maidment; Mays (1994), citados por Segovia; Hang (2000), mencionam que esse é um método empírico para o cálculo da transformação da precipitação em escoamento direto, que surgiu da observação do fenômeno hidrológico em diferentes tipologias de solos, usos e condições antecedentes de umidade. Seu objetivo era estimar o escoamento superficial em pequenas bacias hidrográficas agrícolas considerando as condições de cobertura e tipo de solos, para se analisar a influência dos tratamentos agrícolas e da mudança no uso. Ferrer Juliá et al. (2003) mencionam que posteriormente se desenvolveu sua aplicação para bacias urbanas. 
A precipitação incidente na bacia e a parte desta que corresponde à precipitação efetiva, que produz escoamento ou vazão direta $(\mathrm{Qd})$, pode ser analisada graficamente no hidrograma de vazão. Para padronização e análise dessa precipitação efetiva, se estabeleceu a teoria da Curva Número, um número adimensional de curva, que varia de zero a 100 , em função das tipologias de recobrimento e das características do solo. Para superfícies impermeáveis ou com lâmina de água superficial, a CN é igual a 100 , e para superfícies naturais, com capacidade de armazenamento de água, a CN é menor que 100. Esses valores de CN são tabelados para diferentes tipos de solos e de uso da terra. Reyes (2010), utilizando essa metodologia de $\mathrm{CN}$, comparou três áreas de nascentes (sub-bacias) quanto à capacidade de armazenamento e resposta hidrológica, evidenciando que a bacia com maior área com floresta apresentou maior capacidade de armazenamento e, consequentemente, menor coeficiente de escoamento superficial.

\section{MATERIAL E MÉTODOS}

A bacia hidrográfica do rio Carapá, localizada no Departamento de Canindeyú, região oriental, porção leste do Paraguai, é uma região de aproximadamente 267.000 hectares, distribuídos em 7 municípios. O principal curso de água é o rio Carapá, afluente da margem esquerda do rio Paraná. A região está localizada entre as coordenadas UTM $667.973 \mathrm{~m} \mathrm{E}$, e $772.615 \mathrm{~m} \mathrm{E} ; 7.287 .936 \mathrm{~m} \mathrm{~N}$ e $7.348 .889 \mathrm{~m} \mathrm{~N}$, Fuso 21, e apresenta uma altitude média de $337 \mathrm{~m}$, chegando a $220 \mathrm{~m}$ na margem do lago da represa de Itaipu (Figura 1).

Esta bacia foi dividida pelo Proyecto Carapá Ypoti (2008) em 55 áreas de nascentes, neste trabalho definidas como sub-bacias. Destas, foram tomadas as sub-bacias número 01, 18 e 49 para o presente estudo, as quais estão localizadas nos municípios de Corpus Christi e Francisco Caballero Alvarez. A localização em coordenadas UTM e superfície das sub-bacias são as seguintes:

- Sub-bacia 01: 719.611m E e 729.960m E; 7.321.552m N e 7.335.005m N, Fuso 21; 8.518,81 ha.

- Sub-bacia 18: 732.342m E e 741.333m E; 7.294.414m N e 7.304.644m N, Fuso 21; 5.807,52 ha.

- Sub-bacia 49: 692.757m E e 701.043m E; 7.294.414m N e 7.304.644m N, Fuso 21; 6.578,77 ha.

Os dois municípios apresentam ocupação predominantemente rural. A vegetação autóctone da área da bacia forma parte do denominado Bosque Atlântico Alto Paraná, que corresponde à porção ocidental do Bosque Atlântico Interior (DINERSTEIN et al., 1995).

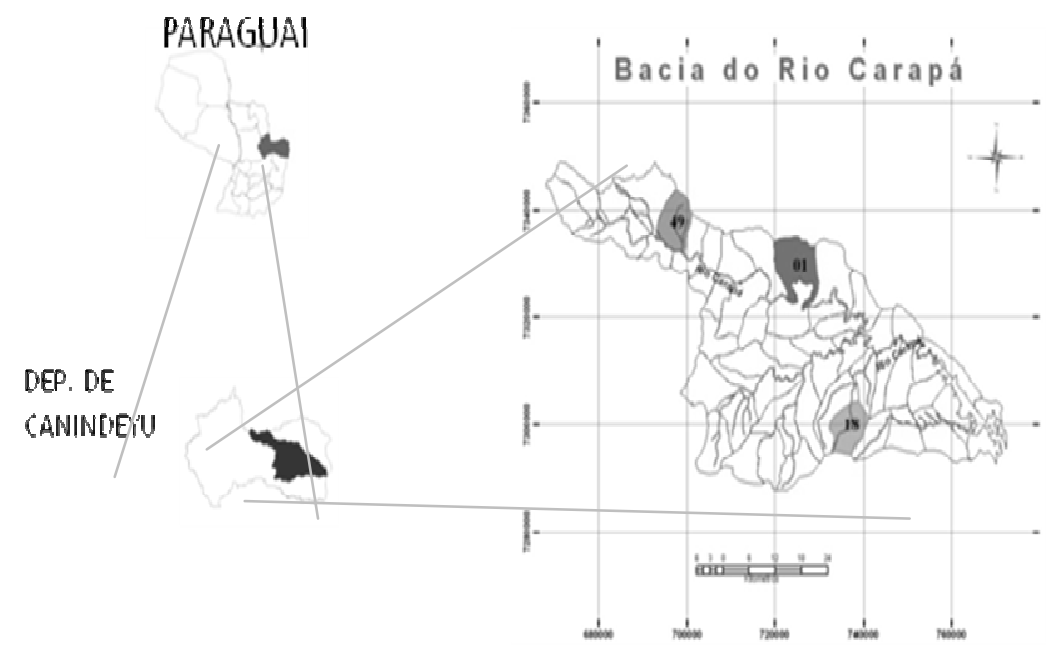

Figura 1. Localização da bacia do rio Carapá, Canindeyú, Paraguai, em destaque divisão em sub-bacias. Figure 1. Location of river basin Carapá, Canindeyú, Paraguay, highlighted the division into sub-basins.

A região apresenta uma proporção importante de solos originados de rochas basálticas, sendo que as restantes são derivadas de arenitos (ATLAS AMBIENTAL, 1995). De acordo com López Gorostiaga et al. (2005), a tabela 1 mostra as classes de solos encontradas nas três sub-bacias e suas correspondentes classes hidrológicas. 
As classes hidrológicas foram determinadas utilizando-se a metodologia (ábaco) da Soil Survey Staff, enquadradas nas classes hidrológicas referenciadas a taxas de infiltração A $\left(>7,6 \mathrm{~mm}^{-1}\right), \mathrm{B}(3,8$ a 7,6 $\left.\mathrm{mm}^{-1}\right), \mathrm{C}\left(1,3\right.$ a $\left.3,8 \mathrm{~mm}^{-1}\right)$ e $\mathrm{D}\left(<1,3 \mathrm{~mm}^{-1}\right)$, em função do conteúdo de argila, silte e areia.

Tabela 1. Classes hidrológicas das classes de solos utilizadas.

Table 1. Classes hydrological soil classes used.

\begin{tabular}{llc}
\hline Bacia & \multicolumn{1}{c}{ Classes de solos } & Classes hidrológicas \\
\hline 01 & Alfisols (Luvissolos) & $\mathrm{C}$ \\
$01-18$ & Entisols (Neossolos litólicos) & $\mathrm{D}$ \\
$01-18-49$ & Oxisols (Latossolos) & $\mathrm{C}$ \\
$01-49$ & Terras mistas & $\mathrm{D}$ \\
\hline
\end{tabular}

O primeiro passo para a realização da análise foi a aquisição das imagens de satélite Landsat 5 TM, órbita 224, cena 77, junto ao INPE, disponível em forma gratuita no site da instituição. Foram adquiridas três imagens da área em estudo dos anos de 1985, 1999 e 2007 (Figura 2), correspondentes à estação seca, para evitar presença de nuvens que ocasionariam erros na classificação. Para o ano de 2007, além das imagens de satélite, contou-se com levantamentos em campo do Proyecto Carapá Ypoti (2008).
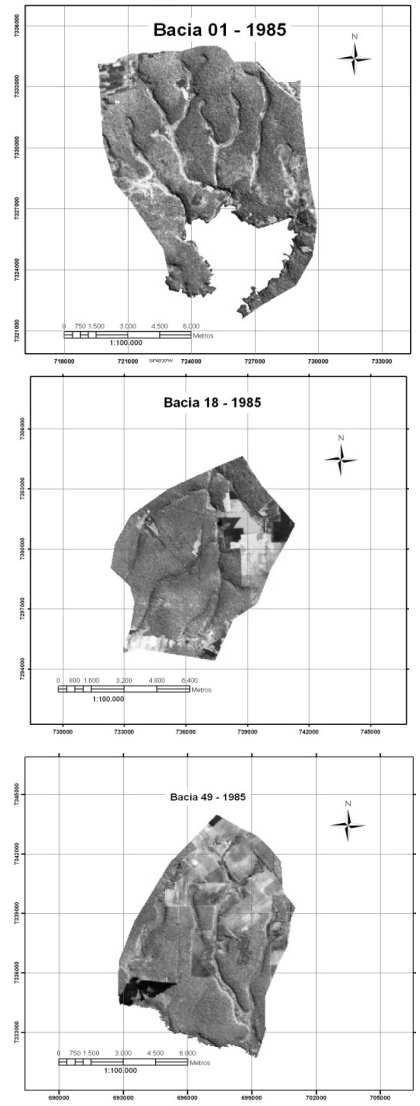
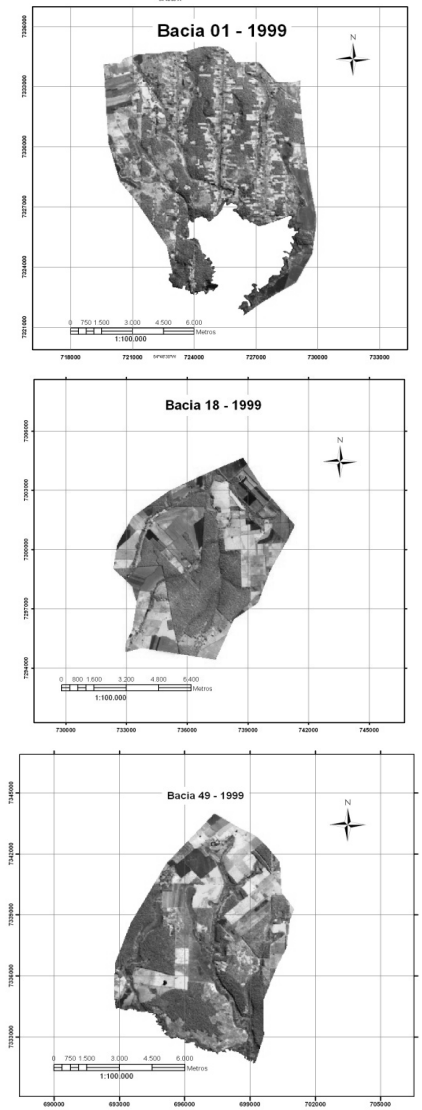
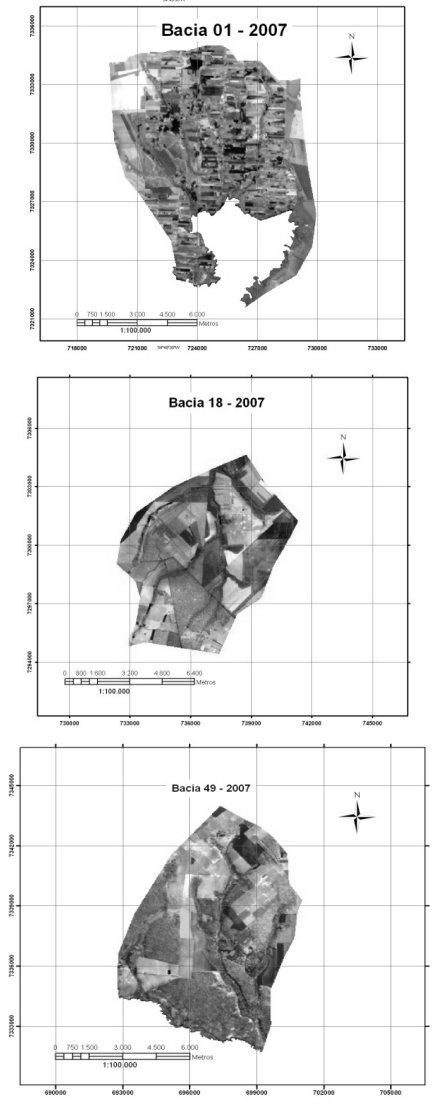

Figura 2. Imagem recortada das sub-bacias 01, 18 e 49 em 1985, 1999 e 2007.

Figure 2. Cropped image of the sub-basin 01, 18 and 49 in 1985, 1999 and 2007.

As tipologias de uso utilizadas foram:

- Floresta nativa: florestas naturais em vários estágios de sucessão.

- Vegetação secundária: vegetação, mas com resposta espectral diferente de floresta nativa. 
- Cultivos: áreas destinadas a cultivos anuais ou campos naturais. Eventualmente podem apresentar resposta espectral de solos expostos, dependendo do estado fenológico das plantas ou da época de preparação do terreno. Referem-se a culturas anuais, pastagens naturais ou implantadas e solos expostos.

- Pastagem: compreende as áreas cobertas por pastagens de acordo com a classificação do Proyeto Carapá Ypoti (2008). A característica desse uso é que representa médios e grandes produtores, que realizam manejos regulares com presença de curvas de nível em grande parte das áreas.

- Alagados (banhados): caracterizam-se pela presença de água, que cobre parte significativa de sua área total, saturando os sedimentos e criando condições de solo encharcado, geralmente em um ambiente redutor, que permite apenas o desenvolvimento de espécies vegetais adaptadas a essas condições. Na classificação de vegetação do Manual Técnico da Vegetação Brasileira (IBGE, 1992, os banhados aparecem como Áreas Pioneiras de Influência Fluvial.

Para o desenvolvimento do trabalho de classificação, empregaram-se técnicas de sensoriamento remoto (registro, segmentação, realce e contraste, entre outros elementos), utilizando-se o software ENVI 4.3. O programa EDISON foi utilizado na segmentação das imagens, para facilitar a separação das classes. A classificação foi realizada nos software do Sistema de Informação Geográfica - SIG (Multispec e ArcGis 9.0).

\section{Análise de números hidrológicos (Curva Número)}

Para a análise de números hidrológicos, foram utilizados os dados resultantes da análise multitemporal para a determinação dos usos do solo nas três bacias nos três períodos. Os dados de uso do solo em formato shapefile correspondem ao levantamento realizado por López Gorostiaga et al. (1995), disponíveis em escala 1:500.000. Foi simulada uma precipitação de $70 \mathrm{~mm}$, com uma duração de 1 hora, objetivando a comparação do comportamento do escoamento superficial nas três bacias nos três períodos. Tomou-se como referência a umidade antecedente normal (NII) para a determinação do número de curva (Tabela 2). As condições de umidade antecedente referem-se a três situações: NI, que implica uma condição do solo seco, mas sem chegar ao ponto de murcha permanente, NII corresponde à condição de umidade normal do solo e NIII corresponde a um solo saturado ou que esteja muito próximo da saturação.

Como não pode ser verificada espectralmente a característica particular de uso para os períodos 1985 e 1999 no que se refere a Cultivo, foi utilizada uma média dos valores de curvas números. As tipologias vinculadas foram: solo lavrado, plantações regulares, plantações de cereais, plantações de legumes ou cultivados, pastagens, campos permanente e chácaras com estradas de terra. Para determinar o valor CN da tipologia Vegetação Secundária nesses períodos, elas foram enquadradas como florestas esparsas de baixa densidade e fragmentos dispersos. Floresta Nativa foi considerada na categoria de florestas naturais. Esse enquadramento foi utilizado nos três períodos. À tipologia Alagados (várzeas úmidas e banhados) utilizada nesta análise, em função de suas características mencionadas por Chow; Maidment; Mays (1994) e Ferrer Juliá et al. (2003), corresponde um valor CN de 100, pois são áreas que apresentam lâminas de água e tudo o que precipita nelas se converte em escoamento.

Segundo a metodologia, o número de curva pode tomar valores variando de 0 (zero) a 100 (cem). O maior número subentende uma menor quantidade de água para saturação do solo e consequentemente uma maior quantidade de água para escoamento superficial (Pe ou Es, que no hidrograma de vazão corresponde ao Qd). Ou seja, em CN = 100 tem-se uma bacia impermeável e toda a precipitação escoa, produzindo escoamento superficial. Mesmo que se enquadrem os solos em relação às classes hidrológicas, é necessário utilizar o número de curva considerando três situações de umidade antecedente (NI, NII, NIII):

- $\quad$ NII corresponde a solo em situação normal de umidade ou de água disponível, em situação de não saturação (capacidade de campo) ou de estresse hídrico (ponto de murcha permanente).

- NI corresponde a solo seco sem, contudo, atingir o ponto de murcha permanente, obtido aplicando-se a seguinte equação:

$$
N I=\frac{4,2 \cdot N I I}{10-0,058 \cdot N I I}
$$

- $\quad$ NIII corresponde a solo saturado ou próximo da saturação ou da capacidade de campo, aplicando-se a seguinte equação:

$$
N I I I=\frac{23 \cdot N I I}{10+0,13 \cdot N I I}
$$

FLORESTA, Curitiba, PR, v. 41, n. 2, p. 243-256, abr./jun. 2011. 
Tabela 2. Valores da curva número para bacias rurais.

Table 2. Values of the curve number for rural basins.

\begin{tabular}{|c|c|c|c|c|c|}
\hline Tipo de uso do solo & Superfície & $\mathbf{A}$ & $\mathbf{B}$ & $\mathbf{C}$ & D \\
\hline \multirow[t]{2}{*}{ Solo lavrado } & Com sulcos retilíneos & 77 & 86 & 91 & 94 \\
\hline & Em fileiras retas & 70 & 80 & 87 & 90 \\
\hline \multirow[t]{3}{*}{ Plantações regulares } & Em curvas de nível & 67 & 77 & 83 & 87 \\
\hline & Terraços em curvas de nível & 64 & 76 & 84 & 88 \\
\hline & Em fileiras retas & 64 & 76 & 84 & 88 \\
\hline \multirow[t]{3}{*}{ Plantações de cereais } & Em curvas de nível & 62 & 74 & 82 & 85 \\
\hline & Terraços em curvas de nível & 60 & 71 & 79 & 82 \\
\hline & Em fileiras retas & 62 & 75 & 83 & 87 \\
\hline \multirow{5}{*}{$\begin{array}{l}\text { Plantações de legumes ou } \\
\text { cultivados (horticultura) }\end{array}$} & Em curvas de nível & 60 & 72 & 81 & 84 \\
\hline & Terraços em curvas de nível & 57 & 70 & 78 & 89 \\
\hline & Pobres & 68 & 79 & 86 & 89 \\
\hline & Normais & 49 & 69 & 79 & 94 \\
\hline & Boas & 39 & 61 & 74 & 80 \\
\hline \multirow[t]{3}{*}{ Pastagens } & Pobres em curvas de nível & 47 & 67 & 81 & 88 \\
\hline & Normais em curvas de nível & 25 & 59 & 75 & 83 \\
\hline & Boas em curvas de nível & 6 & 35 & 70 & 79 \\
\hline \multirow[t]{4}{*}{ Campos permanentes } & Normais & 30 & 58 & 71 & 78 \\
\hline & Esparsos de baixa transpiração & 45 & 66 & 77 & 83 \\
\hline & Normais de média transpiração & 36 & 60 & 73 & 79 \\
\hline & Densos de alta transpiração & 25 & 55 & 70 & 77 \\
\hline \multirow[t]{3}{*}{ Chácaras com estradas de terra } & Normais & 56 & 75 & 86 & 91 \\
\hline & Má conservação & 72 & 82 & 87 & 89 \\
\hline & De superfícies duras & 74 & 84 & 90 & 92 \\
\hline \multirow[t]{4}{*}{ Florestas } & Esparsas, baixa transpiração & 56 & 75 & 86 & 91 \\
\hline & Esparsas & 46 & 68 & 78 & 84 \\
\hline & Densas de alta transpiração & 26 & 52 & 62 & 69 \\
\hline & Normais & 36 & 60 & 70 & 76 \\
\hline
\end{tabular}

FONTE: Tucci et al. (1993).

Fixados os números hidrológicos das distintas áreas de uso do solo existentes na bacia hidrográfica, calcula-se o número de Curva Média em um primeiro momento para cada tipologia de uso do solo e classe hidrológica, e num segundo momento para o conjunto das áreas da bacia, utilizando-se a seguinte equação:

$$
N=\frac{\sum(\text { Nixai })}{A}
$$

Em que: $\mathrm{N}=$ o número de curva média para cada tipologia ou bacia;

$\mathrm{Ni}=$ o número de curva da área de uso do solo relacionado à classe hidrológica $\mathrm{A}, \mathrm{B}, \mathrm{C}, \mathrm{D}$;

$\mathrm{ai}=\mathrm{a}$ área de uso de cada tipologia;

$\mathrm{A}=\mathrm{a}$ área total da bacia hidrográfica.

\section{Capacidade máxima de saturação da bacia (S)}

Representa a capacidade máxima de armazenamento de água, a qual é influenciada pelo tipo de solo, pela umidade inicial antes da precipitação, pela vegetação que sustenta e pelo tratamento e cobertura do solo. Representa, portanto, o potencial máximo de retenção de água que tem o complexo solovegetação. É dado pela equação:

$$
S=\frac{25400}{C N}-254
$$

\section{Conteúdo de chuva abstraída ou encharcamento (Po)}

Po é o limite máximo de umidade a partir do qual começa o escoamento superficial, ou seja, altura mínima de chuva necessária para que se produza o escoamento superficial. No modelo, esta 
abstração, depois de inúmeros experimentos, corresponde a $1 / 5$ (ou $20 \%$ da capacidade máxima do potencial de retenção $S)$, sendo obtido pela relação: $P o(m m)=0,2 S(20 \%$ de $S$ ) ou então pela equação:

$$
P o=\frac{5080}{C N}-50,8
$$

\section{Escoamento superficial real ou precipitação efetiva (Pe)}

Pe (ou Es) é a quantidade de precipitação que produz o escoamento superficial ou vazão direta (Qd) do hidrograma de vazão, ou seja, produz aumento do nível do leito do rio. O total de Pe (Es) ao escorrer pela superfície do solo poderá produzir erosão. Em síntese, é a quantidade de água que sobra depois de certa quantidade de água ser "abstraída" e "infiltrada" na bacia. É dado pela equação:

$$
P e=\frac{\left(P p-\frac{5080}{C N}+50,8\right)^{2}}{P p+\frac{20320}{C N}+203,2}
$$

Em que: $\mathrm{Pp}=$ a precipitação incidente na bacia hidrográfica.

\section{Perdas reais máximas (Pmax)}

É o conteúdo de água que permanece na bacia e posteriormente pode servir como água disponível para os cultivos, abastecer o lençol freático, através da percolação profunda, ou mesmo sofrer processo de evaporação. É dada pela equação:

$$
P_{\text {máx }}=P p-P o-P e
$$

\section{Coeficiente de escoamento superficial (CE)}

O tempo que a água de escoamento superficial demanda para alcançar o leito do rio, que pode ser identificado no hidrograma de vazão (escoamento superficial, sub-superficial e precipitação que incide diretamente na superfície do canal do rio), é considerado como escoamento direto (Qd). O volume do escoamento direto costuma ser comparado com a precipitação incidente na bacia. Assim, surge o conceito de coeficiente de escoamento superficial (CE), que depende do tempo em consideração e que é expresso como:

$$
C E=\frac{P e}{P p}
$$

Em que: $\mathrm{Pe}=\mathrm{o}$ escoamento superficial real ou aporte específico da bacia que alcança o leito do rio e faz com que o nível do rio se eleve;

$\mathrm{Pp}=\mathrm{a}$ precipitação incidente sobre a bacia, sendo comumente expresso em porcentagem (\%).

\section{RESULTADO E DISCUSSÕES}

A Curva Número Média para cada tipologia de uso da terra é calculada em função da cobertura e classe hidrológica do solo (Tabela 3) para cada sub-bacia. Segue exemplo para a sub-bacia 01, ano de 1985.

- NII média para cultivo $=\{(584,63 \times 80)+(0,63 \times 86)+(1,15 \times 80)+(71,13 \times 86)\} \div(584,63+0,63$ $+1,15+71,13)=80,64$

- NII média para vegetação secundária $=\{(883,59 \times 86)+(3,25 \times 91)+(1,52 \times 86)+(101,50 \times 91)\} \div$ $(883,59+3,25+1,52+101,50)=86,49$

- NII média para floresta $=\{(6.670,35 \times 70)+(12,45 \times 76)+(4,18 \times 70)+(184,43 \times 76)\} \div(6670,35+$ $12,45+4,20+84,43)=70,14$

Número de Curva Médio da sub-bacia é dado por $\sum$ Uso(\%) x CNII

- NII média $=(7,72 \times 80,64 / 100)+(11,62 \times 86,49 / 100)+(80,64 \times 70,14 / 100)=72,85$

Aplicando, para a sub-bacia 01, ano 1985, as equações para NI e NII (Tabela 8)

- $\mathrm{NI}$ média $=(4,2 \times 72,85) \div(10-0,058 \times 72,85)=52,98$

- NIII média $(23 \times 72,85) \div(10+0,13 \times 72,85)=86,06$ 
Tabela 3. Área por tipologia de uso do solo na sub-bacia $01 \mathrm{em} 1985$.

Table 3. Surface soil cover of sub-basin 01 in 1985.

\begin{tabular}{lcccc}
\hline Classe hidrológica do solo & Cultivo agrícola & Vegetação secundária & Floresta nativa & Total \\
\hline A & 584,63 & 883,59 & $6.670,35$ & $8.138,57$ \\
B & 0,63 & 3,25 & 12,45 & 16,33 \\
C & 1,15 & 1,52 & 4,18 & 6,85 \\
D & 71,13 & 101,50 & 184,43 & 357,06 \\
& 657,55 & 989,86 & $6.871,41$ & $8.518,81$ \\
\hline
\end{tabular}

O resultado da classificação das três sub-bacias nos período de 1985, 1999 e 2007 são apresentados nas figuras 3, 4 e 5 e nas tabelas 4, 5, 6 e 8, acompanhados dos valores de Curva Número Média de cada uma das tipologias de uso do solo e para cada uma das sub-bacias estudadas.

Procedendo-se da mesma maneira para as sub-bacias 18 e 19, registram-se os dados da tabela $4 \mathrm{e}$ figura 4. Observa-se nessa tabela que no período de 1985 a tipologia de uso predominante nas três subbacias é Floresta Nativa, com valores de $80,66 \%$ na sub-bacia $01,74,25 \%$ na sub-bacia 18 e $61,22 \%$ na sub-bacia 49.

Tabela 4. Superfície e valores de Curva Número médios NII das sub-bacias 01, 18 e 49 em 1985.

Table 4. Surface and Curve Number values averages NII of sub-basins 01, 18 and 49 in 1985.

\begin{tabular}{lccccccccc}
\hline Ano 1885 & \multicolumn{3}{c}{ Bacia 01 } & \multicolumn{3}{c}{ Bacia 18 } & \multicolumn{3}{c}{ Bacia 49 } \\
\hline Usos & ha & $\%$ & $\begin{array}{c}\text { CN } \\
\text { média }\end{array}$ & ha & \% & $\begin{array}{c}\text { CN } \\
\text { média }\end{array}$ & ha & \% & $\begin{array}{c}\text { CN } \\
\text { média }\end{array}$ \\
\hline Cultivo & 657,55 & 7,72 & 80,64 & 807,04 & 13,9 & 80,02 & 1428,67 & 21,72 & 80,76 \\
Vegetação & 989,84 & 11,62 & 86,49 & 689,18 & 11,87 & 86,12 & 1122,49 & 17,06 & 87,42 \\
secundária & & 80,66 & 70,14 & 4311,3 & 74,24 & 70,00 & 4027,61 & 61,22 & 71,02 \\
Floresta nativa & 6871,42 & 100 & & 5807,52 & 100 & & 6578,77 & 100 & \\
\hline Total & 8518,81 & 100 & & 73,31 & & & 75,94 \\
\hline Curva média & & 72,85 & & &
\end{tabular}
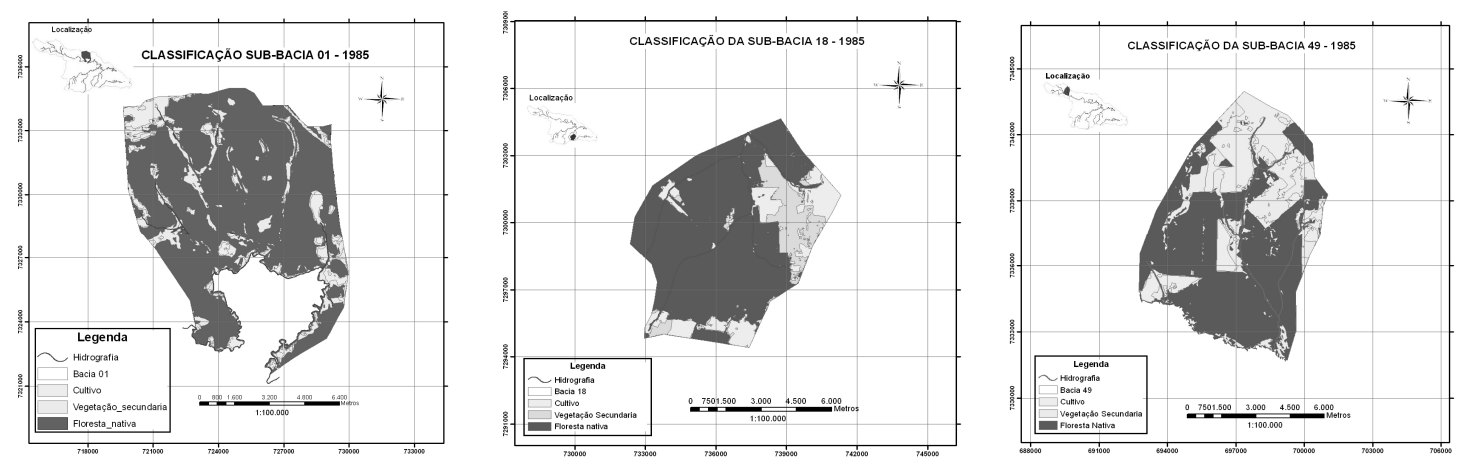

Figura 3. Mapa de uso do solo das sub-bacias 01, 18 e 49 em 1985.

Figure 3. Map of land use sub-basins 01, 18 and 49 in 1985.

No período de 1999 (Tabela 5 e Figura 5), a tipologia de uso predominante na sub-bacia 01 é Cultivo, indicando uma forte diminuição da Floresta Nativa. Na sub-bacia 18, observa-se que a tipologia Vegetação Secundária é a que apresenta maior valor, com $42,7 \%$. Floresta Nativa aparece com uma diminuição acentuada, com 37,24\%. Na sub-bacia 49 também predomina Vegetação Secundária, com $52,51 \%$, seguida de Floresta Nativa, com 33,02\%, marcando tendência nas três áreas com forte indicativo de desmatamento.

Na tabela 6 e figura 6 (2007), encontra-se que a tipologia de uso Cultivo é a que apresenta maior superfície nas sub-bacias 01 e 18 , com valores de $87,03 \%$ e 72,01\%, respectivamente. Nesse período, a sub-bacia 49 apresenta Floresta Nativa como tipologia predominante $(41,08 \%)$. 
Tabela 5. Superfície e valores de Curva Número NII médios das sub-bacias 01, 18 e 49 em 1999.

Table 5. Surface and Curve Number values NII averages of sub-basins 01, 18 and 49 in 1999.

\begin{tabular}{lccccccccc}
\hline Ano 1999 & \multicolumn{3}{c}{ Bacia 01 } & \multicolumn{3}{c}{ Bacia 18 } & \multicolumn{3}{c}{ Bacia 49 } \\
\hline Usos & ha & \% & $\begin{array}{c}\text { CN } \\
\text { média }\end{array}$ & ha & \% & $\begin{array}{c}\text { CN } \\
\text { média }\end{array}$ & ha & \% & $\begin{array}{c}\text { CN } \\
\text { média }\end{array}$ \\
\hline Cultivo & 3401,86 & 39,93 & 80,25 & 1165,21 & 20,06 & 80,00 & 951,84 & 14,47 & 80,74 \\
Vegetação secundária & 1774,85 & 20,83 & 86,21 & 2479,82 & 42,7 & 86,04 & 3454,75 & 52,51 & 87,10 \\
Floresta nativa & 3342,1 & 39,23 & 70,21 & 2162,49 & 37,24 & 70,00 & 2172,18 & 33,02 & 70,85 \\
\hline Total & 8518,81 & 100 & & 5807,52 & 100 & & 6578,77 & 100 \\
\hline Curva média & & 77,54 & & & 78,85 & & & 80,82 \\
\hline
\end{tabular}

Observa-se na tabela 7 que a sub-bacia 01 é a que apresentou maior taxa de desmatamento anual nos três períodos estudados e nas três áreas analisadas, com um valor médio de 3,19\%, seguida da subbacia $18(2,38 \%)$ e da sub-bacia 49 , com $0,71 \%$. Também foi a sub-bacia 01 que apresentou maior taxa linear de desmatamento nos 22 anos compreendidos entre 1985 e 2007, com 69,31\% de diminuição de sua área original. Todas as áreas apresentaram taxa anual de desmatamento menor que a média para a região oriental do Paraguai no período de 1984 a 1991, que, segundo Huespe Fatecha et al. (1994), é de $4,7 \%$ e $4,4 \%$ para o Departamento de Canindeyú.
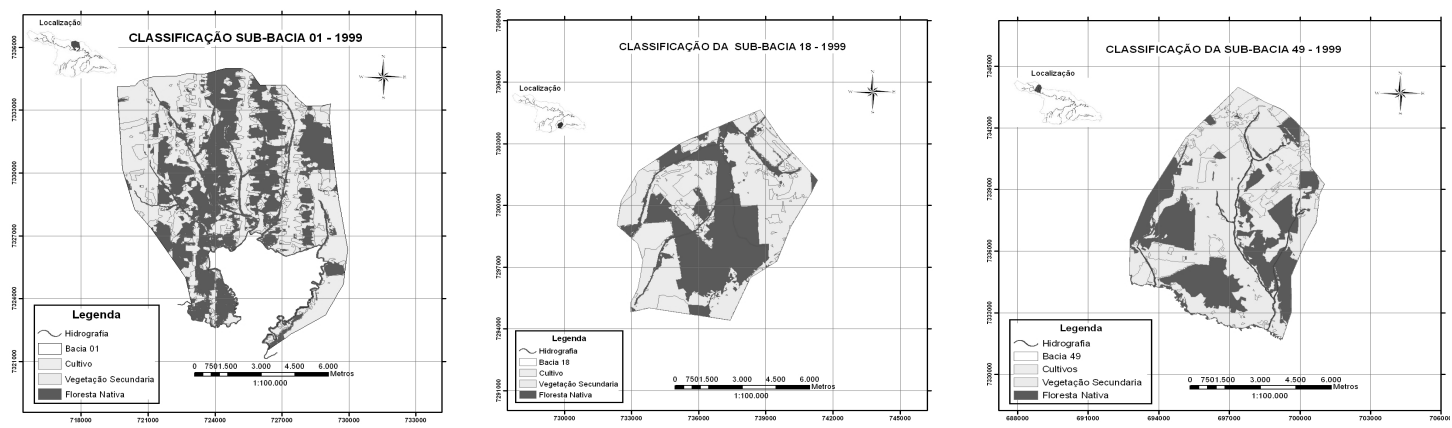

Figura 5. Mapa de uso do solo das sub-bacias 01, 18 e 49 em 1999.

Figure 5. Map of land use sub-basins 01, 18 and 49 in 1999.

Tabela 6. Superfície e valores de Curva Número NII médios das sub-bacias 01; 18 e 49 em 2007.

Table 6. Surface and Curve Number values averages NII of sub-basins 01, 18 and 49 in 2007.

\begin{tabular}{lccccccccc}
\hline Ano 2007 & \multicolumn{3}{c}{ Bacia 01 } & \multicolumn{3}{c}{ Bacia 18 } & \multicolumn{3}{c}{ Bacia 49 } \\
Usos & ha & $\%$ & $\begin{array}{c}\text { CN } \\
\text { média }\end{array}$ & ha & \% & $\begin{array}{c}\text { CN } \\
\text { média }\end{array}$ & ha & \% & $\begin{array}{c}\text { CN } \\
\text { média }\end{array}$ \\
\hline Cultivo & 7414,05 & 87,03 & 84,12 & 4182,24 & 72,01 & 84,02 & 1300,53 & 19,77 & 85,26 \\
Floresta nativa & 967,15 & 11,35 & 70,96 & 1195,23 & 20,58 & 70,00 & 2702,28 & 41,08 & 71,52 \\
Pastagem & 0 & 0 & - & 355,37 & 6,12 & 75,00 & 1978,65 & 30,08 & 75,80 \\
Banhado & 137,6 & 1,62 & 100 & 74,68 & 1,29 & 100 & 597,31 & 9,08 & 100 \\
\hline Total & 8518,8 & 100,00 & & 5807,52 & 100,00 & & 6578,77 & 100,00 \\
\hline Curva média & & 82,88 & & & 80,79 & & & 77,91 \\
\hline
\end{tabular}

A tabela 7 mostra que no período entre 1999 e 2007 foi observado um comportamento negativo de $-3,84 \%$ (taxa anual de $-0,48 \%$ ) do desmatamento, evidenciando um aumento da massa florestal. Esse valor possivelmente pode ser atribuído à resposta espectral da imagem não distinguir perfeitamente a Floresta Nativa com a tipologia Vegetação Secundária.

A tabela 8 apresenta os parâmetros hidrológicos das três sub-bacias nos três períodos com simulação de $70 \mathrm{~mm}$ de precipitação, tendo como $\mathrm{CN}$ média a média ponderada para cada uma das tipologias de uso em relação às classes hidrológicas.

FLORESTA, Curitiba, PR, v. 41, n. 2, p. 243-256, abr./jun. 2011. 

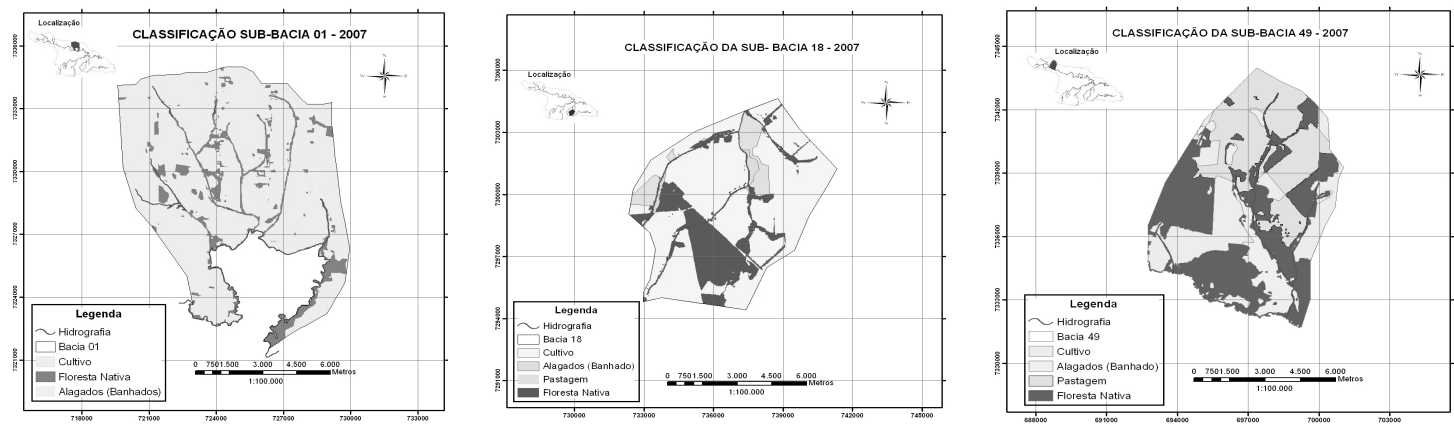

Figura 6. Mapa de uso do solo das sub-bacias 01, 18 e 49 em 2007.

Figure 6. Map of land use sub-basins 01, 18 and 49 in 2007.

Tabela 7. Taxa de desmatamento nas três sub-bacias nos três períodos.

Table 7. Rate of the deforestation rate in the three sub-basins in the three periods.

\begin{tabular}{lccccccc}
\hline \multirow{2}{*}{ Ano } & \multirow{2}{*}{$\begin{array}{c}\text { Período } \\
(\mathbf{a n o s})\end{array}$} & \multicolumn{2}{c}{ Bacia 01 } & \multicolumn{2}{c}{ Bacia 18 } & \multicolumn{2}{c}{ Bacia 49 } \\
\cline { 3 - 8 } & $\begin{array}{c}\text { Desmatamento } \\
(\boldsymbol{\%})\end{array}$ & $\begin{array}{c}\text { Taxa anual } \\
(\boldsymbol{\%})\end{array}$ & $\begin{array}{c}\text { Desmatamento } \\
(\boldsymbol{\%})\end{array}$ & $\begin{array}{c}\text { Taxa anual } \\
(\boldsymbol{\%})\end{array}$ & $\begin{array}{c}\text { Desmatamento } \\
(\boldsymbol{\%})\end{array}$ & $\begin{array}{c}\text { Taxa anual } \\
(\boldsymbol{\%})\end{array}$ \\
\hline 1985 & 14 & 41,43 & 2,95 & 37 & 2,64 & 23,98 & 1,71 \\
1999 & 8 & 27,88 & 3,48 & 16,66 & 2,08 & $-3,84$ & $-0,48$ \\
2007 & 22 & 69,31 & 3,15 & 53,66 & 2,43 & 20,14 & 0,91 \\
Média & & & 3,19 & & 2,38 & & 0,71 \\
\hline
\end{tabular}

As estimativas de CN média da sub-bacia 01 no ano de 1985 apresentam os valores de 52,98 para condição seca (NI), 72,85 para condição normal (NII) e 86,66 para condição de solo saturado (NIII). Tendo como referência os dados de uso do solo da tabela 4, de 7,72\% de Cultivos, 11,62\% de Vegetação Secundária e $80,66 \%$ de Floresta Nativa, observa-se na tabela 8 uma capacidade de saturação de 225,93 mm quando em situação de estiagem ou solos secos (NI), capacidade de saturação de 94,66 mm em situação média de umidade (NII), e 41,16 mm quando em estado de umidade próximo da saturação ou de capacidade de campo.

Observa-se, ainda, que na sub-bacia 01 são necessários 45,08 mm de chuva de "abstração" (Po) em situação de estiagem ou secos (NI), de no mínimo 18,93 mm de chuva para produzir escoamento superficial em situação média de umidade (NII) e de $8,23 \mathrm{~mm}$ quando em estado de umidade próximo da saturação ou de capacidade de campo (NIII).

Em relação ao escoamento real ou precipitação efetiva $(\mathrm{Pe})$, a sub-bacia 01, com esse uso de solo, em situação de umidade média (normal) produzirá escoamento superficial $(\mathrm{Pe}) \mathrm{de} 17,90 \mathrm{~mm} \mathrm{em}$ situação média de umidade (NII), em situação de estiagem ou secos (NI) apenas 2,44 mm e 24,70 mm quando em estado de umidade próximo da saturação ou de capacidade de campo (NIII), para uma precipitação incidente (Pp) simulada de $70 \mathrm{~mm}$.

Essa mesma sub-bacia 01 permitiria uma perda máxima de água da ordem de $33,17 \mathrm{~mm} \mathrm{em}$ situação média de umidade (NII), de 22,44 mm quando em situação de estiagem ou secos (NI) e 24,70 mm quando em estado de umidade próximo da saturação ou de capacidade de campo (NIII), com precipitação de $70 \mathrm{~mm}$.

Ainda, essa sub-bacia, no ano de 1985, apresentou um coeficiente de escoamento superficial (CE) de 3,55\% em situação de estiagem ou secos (NI), 25,56\% em condição média de umidade (NII) e $52,96 \%$ em estado de umidade próximo da saturação ou de sua capacidade de campo (NIII), para uma precipitação incidente de $70 \mathrm{~mm}$.

Fazendo uma análise do comportamento hidrológico das três sub-bacias, com ênfase no parâmetro de coeficiente de escoamento superficial (CE), observa-se que no período 1985 a sub-bacia 49 obteve o valor mais alto nas três situações de umidade antecedente $(\mathrm{NI}=6,42 ; \mathrm{NII}=30,88 ; \mathrm{NIII}=57,86)$ com relação à sub-bacia $18(\mathrm{NI}=3,91$; NII $=26,33$; NIII $=53,68)$, ficando por último a sub-bacia 01 $(\mathrm{NI}=3,55 ; \mathrm{NII}=25,56 ; \mathrm{NIII}=52,96)$. 
Tabela 8. Curvas número e parâmetros hidrológicos para as três sub-bacias nos anos de 1985, 1999 e 2007.

Table 8. Curve number and hydrological parameters for the three sub-basins in the years 1985, 1999 and 2007.

\begin{tabular}{|c|c|c|c|c|c|c|}
\hline & $\begin{array}{c}\text { CN } \\
\text { Média }\end{array}$ & $\begin{array}{l}\text { Armazenamento } \\
\text { S (mm) }\end{array}$ & $\begin{array}{c}\text { Abstração } \\
\text { Po (mm) }\end{array}$ & 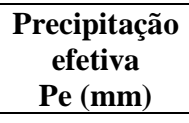 & $\begin{array}{c}\text { Perdas } \\
\text { máximas } \\
\text { Pmax }(\mathbf{m m}) \\
\end{array}$ & $\begin{array}{c}\text { Coeficiente de } \\
\text { escoamento } \\
\text { CE }(\%) \\
\end{array}$ \\
\hline \multicolumn{7}{|l|}{ Ano 1985} \\
\hline \multicolumn{7}{|l|}{ Bacia 01} \\
\hline Seco $(\mathrm{NI})$ & 52,98 & 225,39 & 45,08 & 2,48 & 22,44 & 3,55 \\
\hline Normal (NII) & 72,85 & 94,66 & 18,93 & 17,90 & 33,17 & 25,56 \\
\hline Saturado (NIII) & 86,06 & 41,16 & 8,23 & 37,07 & 24,70 & 52,96 \\
\hline \multicolumn{7}{|l|}{ Bacia 18} \\
\hline Seco (NI) & 53,57 & 220,15 & 44,03 & 2,74 & 23,23 & 3,91 \\
\hline Normal (NII) & 73,31 & 92,46 & 18,49 & 18,43 & 33,08 & 26,33 \\
\hline Saturado (NIII) & 86,34 & 40,20 & 8,04 & 37,58 & 24,38 & 53,68 \\
\hline \multicolumn{7}{|l|}{ Bacia 49} \\
\hline Seco (NI) & 57,00 & 191,65 & 38,33 & 4,49 & 27,18 & 6,42 \\
\hline Normal (NII) & 75,94 & 80,49 & 16,10 & 21,62 & 32,28 & 30,88 \\
\hline Saturado (NIII) & 87,89 & 35,00 & 7,00 & 40,50 & 22,50 & 57,86 \\
\hline \multicolumn{7}{|l|}{ Ano 1999} \\
\hline \multicolumn{7}{|l|}{ Bacia 01} \\
\hline Seco $(\mathrm{NI})$ & 59,19 & 175,14 & 35,03 & 5,82 & 29,15 & 8,32 \\
\hline Normal (NII) & 77,54 & 73,56 & 14,71 & 23,72 & 31,56 & 33,89 \\
\hline Saturado (NIII) & 88,82 & 31,98 & 6,40 & 42,32 & 21,28 & 60,46 \\
\hline \multicolumn{7}{|l|}{ Bacia 18} \\
\hline Seco (NI) & 61,03 & 162,18 & 32,44 & 7,06 & 30,50 & 10,09 \\
\hline Normal (NII) & 78,85 & 68,11 & 13,62 & 25,53 & 30,85 & 36,47 \\
\hline Saturado (NIII) & 89,56 & 29,61 & 5,92 & 43,82 & 20,25 & 62,60 \\
\hline \multicolumn{7}{|l|}{ Bacia 49} \\
\hline Seco (NI) & 63,89 & 143,56 & 28,71 & 9,22 & 32,07 & 13,17 \\
\hline Normal (NII) & 80,82 & 60,30 & 12,06 & 28,39 & 29,55 & 40,56 \\
\hline Saturado (NIII) & 90,64 & 26,22 & 5,24 & 46,10 & 18,66 & 65,85 \\
\hline \multicolumn{7}{|l|}{ Ano 2007} \\
\hline \multicolumn{7}{|l|}{ Bacia 01} \\
\hline Seco (NI) & 67,03 & 124,92 & 24,98 & 11,92 & 33,09 & 17,03 \\
\hline Normal (NII) & 82,88 & 52,47 & 10,49 & 31,62 & 27,88 & 45,18 \\
\hline Saturado (NIII) & 91,76 & 22,81 & 4,56 & 48,52 & 16,92 & 69,32 \\
\hline \multicolumn{7}{|l|}{ Bacia 18} \\
\hline Seco (NI) & 63,85 & 143,83 & 28,77 & 9,19 & 32,05 & 13,12 \\
\hline Normal (NII) & 80,79 & 60,41 & 12,08 & 28,35 & 29,57 & 40,50 \\
\hline Saturado (NIII) & 90,63 & 26,26 & 5,25 & 46,06 & 18,69 & 65,80 \\
\hline \multicolumn{7}{|l|}{ Bacia 49} \\
\hline Seco (NI) & 59,70 & 171,48 & 34,30 & 6,15 & 29,55 & 8,79 \\
\hline Normal (NII) & 77,91 & 72,02 & 14,40 & 24,22 & 31,38 & 34,60 \\
\hline Saturado (NIII) & 89,02 & 31,31 & 6,26 & 42,74 & 21,00 & 61,06 \\
\hline
\end{tabular}

Quanto ao coeficiente de escoamento superficial com a cobertura de Floresta Nativa nas três áreas no ano de 1985, observa-se que a sub-bacia 49 é a que apresenta uma menor cobertura da tipologia Floresta Nativa $(61,22 \%)$ e, na situação de umidade NII, o maior coeficiente de escoamento (CE), de $57,86 \%$, seguida da sub-bacia $18(52,68 \%)$ e da sub-bacia $01(52,96 \%)$. Nesse caso, o menor valor de coeficiente de escoamento superficial corresponde à área que apresenta maior cobertura de Floresta Nativa.

A mesma tendência é observada no período 1999, com valores de coeficiente de escoamento superficial (CE) maiores na sub-bacia 49 nas três situações de umidade antecedente $(\mathrm{NI}=13,17$;

FLORESTA, Curitiba, PR, v. 41, n. 2, p. 243-256, abr./jun. 2011. 
$\mathrm{NII}=40,56 ; \mathrm{NIII}=65,85)$, em comparação com a sub-bacia $18(\mathrm{NI}=10,09 ; \mathrm{NII}=36,47 ; \mathrm{NIII}=62,60)$ e a sub-bacia $01(\mathrm{NI}=8,32 ; \mathrm{NII}=33,89$; $\mathrm{NIII}=60,46)$.

No período de 2007, observa-se uma inversão na ordem de sub-bacias mais propensas à erosão, pois os maiores valores de coeficiente de escoamento superficial (CE) nas três situações de umidade antecedente são encontrados na sub-bacia $01(\mathrm{NI}=17,03$; NII $=45,18$; NIII $=69,32)$, seguida da subbacia $18(\mathrm{NI}=13,12 ; \mathrm{NII}=40,50 ; \mathrm{NIII}=65,80)$ e por último a sub-bacia $49(\mathrm{NI}=8,79 ; \mathrm{NII}=34,60$; $\mathrm{NIII}=61,06)$. Na correlação com o uso do solo nesse período, encontra-se a tipologia Floresta Nativa ocupando $33,02 \%$ na sub-bacia $49,20,58 \%$ na sub-bacia 18 e 11,35\% na sub-bacia 01 . Além disso, a tipologia Cultivos apresenta um aumento considerável na sub-bacia 01, com um valor de $87,03 \%$, sendo que na sub-bacia 18 essa tipologia ocupa $72,01 \%$ e na sub-bacia 49 ocupa 19,77\%.

Com relação ao coeficiente de escoamento superficial com a cobertura de Floresta Nativa nas três áreas no ano de 1985, observa-se que a sub-bacia 49 é a que apresenta uma menor cobertura da tipologia Floresta Nativa $(61,22 \%)$ e, na situação de umidade NII, o maior coeficiente de escoamento (CE) de 57,86\%, seguido da sub-bacia $18(52,68 \%)$ e da sub-bacia $01(52,96 \%)$. Neste caso, o menor valor de coeficiente de escoamento superficial corresponde à área que apresenta maior cobertura de Floresta Nativa. A mesma tendência é observada no período 1999, com valores de coeficiente de escoamento superficial (CE) maiores na sub-bacia 49 nas três situações de umidade antecedente $(\mathrm{NI}=13,17 ; \mathrm{NII}=40,56 ; \mathrm{NIII}=65,85)$ em comparação com a sub-bacia $18(\mathrm{NI}=10,09 ; \mathrm{NII}=36,47$; $\mathrm{NIII}=62,60)$ e com a sub-bacia $01(\mathrm{NI}=8,32 ; \mathrm{NII}=33,89 ; \mathrm{NIII}=60,46)$.

No período de 2007 observa-se uma inversão na ordem de sub-bacias mais propensas à erosão, pois os maiores valores de coeficiente de escoamento superficial (CE) nas três situações de umidade antecedente são encontrados na sub-bacia $01(\mathrm{NI}=17,03$; NII $=45,18$; NIII $=69,32)$ seguida da subbacia $18(\mathrm{NI}=13,12 ; \mathrm{NII}=40,50 ; \mathrm{NIII}=65,80)$ e por último da sub-bacia $49(\mathrm{NI}=8,79 ; \mathrm{NII}=34,60$; $\mathrm{NIII}=61,06)$. Ao correlacionar com o uso do solo nesse período, encontra-se a tipologia Floresta Nativa ocupando $33,02 \%$ na sub-bacia $49,20,58 \%$ na sub-bacia 18 e 11,35\% na sub-bacia 01 . Além disso, a tipologia Cultivos apresenta um aumento considerável na sub-bacia 01 , com um valor de $87,03 \%$, sendo que na sub-bacia 18 essa tipologia ocupa 72,01\% e na sub-bacia 49 ocupa 19,77\%.

\section{CONCLUSÕES E RECOMENDAÇÕES}

- A metodologia da Curva Número é um bom instrumento para análise da capacidade de armazenamento de água de uma bacia, por internalizar em sua concepção o hidrograma de vazão, mais especificamente, o volume de água incidente e de deságue da bacia hidrográfica. Limita-se, portanto, a análise da recarga, armazenamento e descarga da bacia, mantendo-se a relação de unidade de volume da precipitação incidente. Entretanto há questões técnicas que ainda estão em processo de investigação, como, por exemplo, a limitação do uso de apenas quatro classes hidrológicas e pouca inferência quanto às classes de declividade do solo.

- Especificamente em relação aos resultados deste artigo, tem-se que o coeficiente de escoamento superficial nas três situações de umidade antecedente da sub-bacia 49 no período de 1985 e 1999 foi o mais alto e o que indica maior possibilidade de degradação nessa área. No período de 2007, o coeficiente de escoamento superficial nas três situações de umidade antecedente da sub-bacia 01 foi o mais alto, indicando maior possibilidade de degradação na área por conta da ação da erosão hídrica. A utilização de três sub-bacias para a análise dificultou bastante o trabalho, em vista das inúmeras informações que foram processadas.

- É recomendável, para futuros trabalhos, diminuir o número de áreas e trabalhar com sub-bacias menores. A utilização de levantamentos de solos em escala de 1:500.000 proporcionou informação muito generalizada das áreas de estudo, recomendando-se para futuras investigações se trabalhar com levantamentos que tenham escalas de mapeamento menores, com o objetivo de obter informações mais precisas.

\section{REFERÊNCIAS}

ATLAS AMBIENTAL DE LA REGIÓN ORIENTAL DEL PARAGUAY. San Lorenzo: Carrera de Ingeniería Forestal (U.N.A.)/GTZ, 1995. v. 2. 
CARDOZO, C. A.; TEIXEIRA, H. C.; SOARES, C. P.; MARTINS, S. V. Caracterização morfométrica da bacia hidrográfica do rio Debossan, Nova Friburgo, RJ. Revista Árvore, Viçosa, v. 30, n. 2, p. 241 - 248, 2006.

CHIARANDA, R. Usos da terra e avaliação da capacidade potencial de armazenamento de água da bacia do rio Cuiabá, MT. 362 p. Tese (Doutorado em Engenharia Florestal) - Setor de Ciências Agrárias, Universidade Federal do Paraná, Curitiba, 2002.

CHOW, V. T.; MAIDMENT, D. R.; MAYS, L. Hidrología aplicada. Santa Fé de Bogotá: McGraw-Hill Interamericana, 1994.

DINERSTEIN, E.; OLSON, D. M.; GRAHAM, D. J.; WEBSTER, A. L.; PRIM, S. A.; BOOKBINDER, M. P. E.; LEDEC, G. A conservation assessment of the terrestrial ecoregions of Latin America and the Caribbean. Washington, DC: International Bank for Reconstruction and Development/World Bank. 1995.

FERRER JULIÁ, M.; BLANCO, J.; RAMÍREZ, J. Análisis de nuevas fuentes de datos para la estimación del parámetro número de curva: perfiles de suelos y teledetección. In: CONGRESO DE INGENIERÍA CIVIL, TERRITORIO Y MEDIO AMBIENTE, 3. 2003. León. Disponível em: <http://www.ciccp.es/biblio_digital/Icitema_III/congreso/pdf/040205.pdf>. Acesso em: 22/11/2009.

HUESPE F. H.; SPINZI, M. L.; CURIEL, M. V.; BURGOS, S.; RODAS, I. O. Uso de la tierra y deforestación en la región oriental del Paraguay, Periodo 1984-1991. Vol. 1, San Lorenzo: UNA/FIA/CIF. 1994.

JENSEN, J. R. Digital change detection. In: sensing perspective. 2. ed. Prentice Hall. 1996. p. 257 - 279.

Introductory digital image processing: A remote

NOVO, E. de M. Sensoriamento remoto: princípios e aplicações. 2. ed. São José dos Campos: Edgar Blücher. 1992.

PROYECTO CARAPA YPOTI. Informe de gestión. Periodo 2004-2008. Asunción, 2008.

REYES, C. D. R. Análise das características hidrológicas de três sub-bacias do rio Carapá (Canindeyú, Paraguai) em função das mudanças da cobertura vegetal em três diferentes épocas. 125 f. Dissertação (Mestrado em Engenharia Florestal) - Setor de Ciências Agrárias, Universidade Federal do Paraná, Curitiba, 2010.

SEGOVIA, M. A.; HANG, M. V. Estimación del valor del número de curva (CN) a través de imágenes satelitales Landsat 5 - TM. Disponível em: 〈http://arandu.org.ar/pub/segovia-hang.pdf〉. Acesso em: 03/09/2009.

TONELlO, K. C. Análise hidroambiental da bacia hidrográfica da Cachoeira das Pombas, Guanhães, MG. 69 f. Dissertação (Mestrado em Ciência Florestal) - Universidade Federal de Viçosa, Viçosa, 2005.

TUCCI, C. E. M. (org.). Hidrologia: ciência e aplicação. Porto Alegre: Editora da Universidade (UFRGS), ABRH: EDUSP. v. 4. 1993.

VILLELA, S. M.; MATTOS, A. Hidrologia aplicada. São Paulo: McGraw-Hill do Brasil, 1975. 
FLORESTA, Curitiba, PR, v. 41, n. 2, p. 243-256, abr./jun. 2011

Riveros Reys, C. D.; Rizzi, N. E.; Araki, H 